\title{
GITANOS: HISTORIA DE UNA MIGRACIÓN
}

\section{JOSÉ CABANES HERNÁNDEZ}

Sociólogo.

\section{LUZZ VERA GARCÍA}

Trabajadora Social de la Asociación P.G. ARAKERANDO. Alicante.

\section{MARÍA ISABEL BERTOMEU MARTÍNEZ}

Trabajadora Social de la Asociación P.G. ARAKERANDO. Alicante.

\author{
"Esta es la vida del emigrante \\ del vagabundo, del sueño errante. \\ Coge tu vida en tu pañuelo. \\ Con tu pobreza tira pa'alante"
}

CELTAS CORTOS

\section{INTRODUCCIÓN}

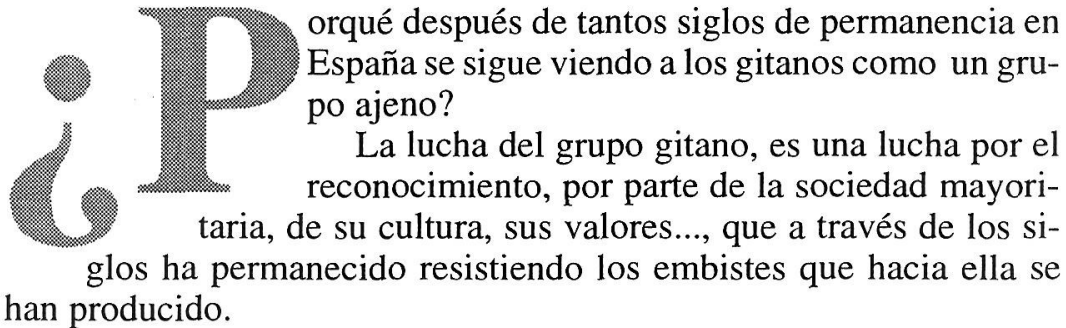

Actualmente, con la entrada de inmigrantes marroquíes y de otros países africanos en nuestro país, migraciones principalmente socioeconómicas, se sigue obviando a los gitanos como ciudadanos españoles, incluyéndolos aún en muchos casos dentro de los grupos de inmigrantes.

En este artículo pretendemos recoger su larga migración desde su origen hasta sus casi seiscientos años de asentamiento en España. Larga historia de rechazo y racismo.

Su conocimiento tal vez pueda servir para ver las posibles causas y consecuencias que pueden tener las actitudes y las políticas de aniquilación para poderlas trasladar a la actualidad.

A pesar de muchos siglos de historia, nuestra actitud con los «diferentes», y sobre todo con los inmigrantes de los países pobres, no ha cambiado tanto como nosotros exigimos que cambien estos. Nuestros estereotipos siguen funcionando. 


\section{UNA LARGA MIGRACIÓN DESDE LA INDIA}

Los gitanos son sin duda los inmigrantes mas antiguos que residen en España, conservando todavía una cultura propia bien diferenciada de la cultura mayoritaria.

Si bien es cierto que la mayor parte de los gitanos residentes en nuestro país son ciudadanos españoles, también lo es el sentimiento apátrida del pueblo gitano como tal, no demostrando un apego hacia ningún lugar establecido en base a unas fronteras políticas

Existen diversas teorías sobre el origen de los gitanos, si bien las más aceptadas en la actualidad son aquellas que los sitúan en el Noroeste de la India, básandose en estudios lingüísticos del Romanó ${ }^{1}$. Se desconoce cuáles fueron las causas que motivaron el éxodo de los gitanos, ni en qué fechas concretas se inició, pero sí existen diversos datos sobre su estancia por distintos países europeos, siempre viajando en pequeños grupos de entre 30 y 150 personas, lo que hace pensar que pudiera tratarse de familias extensas que viajaban agrupados.

«Los gitanos se presentan en Europa como un grupo nómada que se verá obligado a entrar en conflicto con los pueblos sedentarios. Estamos, en cierto modo, ante la dicotomía tradicional pastor o nómada versus, agricultor o sedentario, casi tan antigua como la propia historia del hombre. El nómada representa al grupo que necesita desplazarse para sobrevivir. El sedentario ha logrado adecuar su entorno a sus propias necesidades y controlar a su favor el medio en el que le ha tocado vivir. El nómada se ve obligado todavía, al menos en parte, a una actitud depredadora. El sedentario es esencialmente productivo, al menos en principio, y recela de cualquier visitante con movilidad. Son dos actitudes mentales y dos tipos de economía que necesariamente están abocados al enfrentamiento ${ }^{2} . »$

\section{LA LLEGADA A ESPAÑA}

La llegada de los primeros grupos de gitanos a España se sitúa alrededor de 1425, coincidiendo con un momento histórico en que se tiende a lograr la unificación tanto política como religiosa de lo que ahora es España. Lo que dará lugar a la expulsión de los últimos musulma-

1 Según Teresa SAN ROMÁN, en "La diferència inquietant: velles i noves estratégies culturals dels gitunos". Alta Falla. BCN, 1994, el romanó es el idioma utilizado por los gitanos en gran parte de Europa. Se trata de una lengua neoaria derivada del sanscrito, de la cual el kaló, utilizado por los gitanos españoles, es un dialecto.

2 SÁNCHEZ ORTEGA, Ma Elena y SAN ROMÁN, Teresa "Entre la marginación y el racismo. Reflexiones sobre la vida de los gitanos". Madrid, 1986. p. 19. 
nes, y posteriormente también de los judíos y moriscos. Paradójicamente, ésta no fue la política que se aplicó (al menos en un primer momento) con los gitanos.

A su llegada a España los grupos de gitanos se presentan como peregrinos que vienen a visitar la tumba del apóstol Santiago, lo que les facilita su tránsito y estancia por diversos lugares de la península. Sin embargo, esta situación privilegiada no duraría mucho tiempo, y ya en 1499 los Reyes Católicos dictan la Primera Pragmática contra ellos:

«Los egipcianos y caldereros extranjeros, durante los sesenta días siguientes al pregón, tomen asiento en lugares y sirvan a señores que les den lo que hubieres menester y no vaguen juntos por los reinos; o que al cabo de sesenta días salgan de España, so pena de cien azotes y destierro la primera vez y que les corten las orejas y tornen a desterrar la segunda vez que fueren hallados».

A ésta, seguirían una serie de pragmáticas a lo largo de varios siglos encaminadas a conseguir no sólo el asentamiento de los gitanos, sino el que abandonasen sus oficios tradicionales, su forma de vestir, su manera de hablar, llegando incluso a prohibir los matrimonios entre gitanos. El incumplimiento de estas leyes conllevaba la persecución, expulsión e incluso la muerte.

Como se puede ver se trata de una política de asimilación encaminada a que dejasen de ser gitanos. Esta política de asimilación forzosa choca con la de expulsión que se aplicaban en casi toda Europa ${ }^{3}$. Como consecuencia se produce un importante número de gitanos que se sedentarizan en España mientras en otros países las expulsiones les obligaban a continuar por los caminos.

La explicación al porqué de esa política de asimilación en España la encontramos en un documento del Consejo de Marzo de 1633, en época de Felipe IV, que dice:

"No parece conveniente expedirlos (a los gitanos) porque la despoblación en que se hallan estos Reinos después que salieron los moriscos, y la que causan las necesidades presentes, no puede sufrir ninguna evacuación por pequeña que sea"'.

\section{EL HISTÓRICO INTENTO DE ASIMILACIÓN}

El fracaso de este intento fagocitario de los gitanos, conseguido sólo de una forma parcial, desembocó como «solución definitiva al

3 Sobre las políticas aplicadas a los gitanos en toda Europa. Véase J.P. LIÉGEOIS, "Gitanos itinerantes". Madrid, 1987.

4 SAN ROMÁN, T. Op. Cit. p. 196. 
problema gitano» en la Pragmática de Fernando VI en 1749. El 30 de julio de ese año se realizó una gigantesca redada que afecta a una gran parte de los gitanos de España.

Previamente a esta redada se habían establecido 75 poblaciones donde se reasentó a los gitanos, según las normativas en proporción de una familia gitana por cada mil familias castellanas. En 1746 ya existía un censo de gitanos en el que constaban 881 familias gitanas en 54 de las 75 poblaciones. Esas fueron las familias objeto de la redada, precisamente las más accesibles, es decir, aquellas que, a costa de grandes esfuerzos y múltiples dificultades, habían conseguido establecerse en algún sitio y ser afectadas en el ejercicio de alguna ocupación. Su captura lleva implícito el embargo y la subasta de sus bienes para atender los gastos de la propia redada.

Tras la redada serían separados los hombres de las mujeres. Estas irán destinadas a «depósitos», mezcla de cárcel, cuartel y fábrica, donde tendrán que trabajar para mantener sus gastos de vestido y alimentación. Por su parte, los hombres fueron destinados a trabajar a los arsenales ${ }^{5}$.

La aplicación de esta Pragmática de encarcelación de los gitanos, que estaría vigente hasta 1765 , supuso un duro revés al proceso de sedentarización iniciado por los gitanos en España ya que:

1. Las familias a las que se detenía eran aquellas con domicilio fijo conocido, como decíamos, las que habían conseguido establecerse y ser aceptadas en el ejercicio de alguna ocupación.

2. Las familias que consiguieron escapar retomaron el nomadismo que años antes debían haber abandonado.

3. Aquellos gitanos que en 1746 seguían practicando el nomadismo, verían reforzada su posición evitando asentarse en ningún lugar por peligro de ser detenidos.

En 1783, reinando ya Carlos III, éste adoptó una perspectiva sobre lo que se había dado en llamar «el problema gitano», totalmente diferente a la de sus antecesores, aunque, como se podía apreciar, las diferencias son de forma pero no de fondo, en el cual sigue pesando la «necesaria asimilación».

Carlos III declara que los gitanos deben tener capacidad para elegir libremente sus propias ocupaciones, y también para poder fijar su domicilio donde mejor les conviniera, a excepción de los Sitios Reales. En conjunto la declaración venía a afirmar que los gitanos tenían derecho a disfrutar, por primera vez en la historia de España, de una situación igualitaria con respecto al resto de los Españoles. «Únicamente se les pedía el cumplimiento de tres condiciones: que abandonasen su

5 Sobre esta redada es interesante consultar el libro de GÓMEZ ALFARO, A. "La gran redada de gitanos". Madrid, 1993. 
peculiar forma de vestir y adornarse, que no hablasen el caló ni en público ni ostentosamente; y que permaneciesen asentados permanentemente en un lugar, abandonado la vida de errante. No se preveía tomar medidas drásticas contra ellos si no cumplían esos requisitos, pero se amenazaba con llevar a un orfelinato a cualquier gitano menor de dieciséis años de quien se supiera que sus padres eran nómadas para que en estos centros estatales aprendieran un oficio ${ }^{6}$.

Con la primera Constitución Española de 1812 se da un paso definitivo para el fortalecimiento de la situación jurídica de los gitanos en el país. Con anterioridad, para que un individuo pudiera ser considerado ciudadano español, tenía que estar vinculado a través de su residencia a un lugar, de tal manera que las personas que llevaban una vida errante no se consideraban españolas. Esta Constitución declaraba que cualquier persona nacida en el país era española. Dura "ley de extranjería" (la de la historia de los gitanos españoles), que les había hecho pasar casi trescientos años en el país para poder conseguir la nacionalidad.

A pesar de su nueva situación política de españoles, lo cierto es que los gitanos han seguido teniendo un trato "especial" en la práctica y, en algunos casos, también en la teoría, como muestran los artículos $4 .^{\circ}$ y 5..$^{\circ}$ del Reglamento de la Guardia Civil de 14 de mayo de 1943, vigentes hasta 1978:

«Artículo 4. ${ }^{\circ}$

Se vigilará escrupulosamente a los gitanos, cuidando mucho de reconocer todos los documentos que tengan, observar sus trajes, averiguar su modo de vivir y cuanto conduzca a formar una idea exacta de sus movimientos y ocupaciones, indagando el punto al que se dirigen en sus viajes y el objeto de ellos.

Artículo 5. ${ }^{\circ}$

Como esta clase de gente no tiene por lo general residencia fija, se traslada con frecuencia de un punto a otro en que sean desconocidos, conviene tomar de ellos todas las noticias necesarias para impedir que cometan robos de caballerías o de otra especie ${ }^{7} . »$

\section{LA MIGRACIÓN A LAS GRANDES URBES}

Durante la segunda mitad de este siglo, y coincidiendo con la expansión económica de los años sesenta, se producen una serie de migraciones campo-ciudad en todo el estado español, de las cuales no

7 BLAY, F. et alt.: Propuesta para un trabajo intercultural en la escuela. Fichas de trabajo. Valencia, 1989. p. 42. 
quedan exentos los gitanos. Durante este proceso, muchas familias gitanas que hasta ese momento residían en zonas rurales, o bien practicaban un seminomadismo en ellas, desempeñando actividades cada vez más en deshuso (compra-venta de animales, cestería, etc.), marchan a la gran ciudad en busca de nuevas oportunidades. Posteriormente, y a medida que va avanzando la década, la mayor parte de este colectivo gitano emigrado a la gran ciudad, se ve sometido a un implacable proceso de concentración que crea aglomeraciones densísimas sólo con gitanos ${ }^{8}$.

Durante los años sesenta y principios de los setenta, muchos gitanos pasaron a ocupar puestos de trabajo en la industria, la construcción y el sector servicios, con el cambio económico y psicológico que esta nueva situación suponía tanto para ellos como para sus familias".

En cualquier caso, la mayor parte de los gitanos no pudieron, o no quisieron, entrar en ese tipo de trabajos asalariados, manteniéndose en una economía marginal (chatarrería, recogida de cartón, espectáculos, etc.), que si bien no da seguridad, sí aportaba en muchos casos el mismo o mayor beneficio económico (tengamos en cuenta que los puestos que se daba a los gitanos solían ser los más bajos), al tiempo que podían seguir conservando su libertad (no estaban bajo las órdenes de un "payo"), se distribuían su tiempo de trabajo (por ejemplo, pueden dejar el trabajo durante una semana para asistir a una boda en otro punto de España, o pasar las navidades), permitía una explotación familiar del trabajo, con la participación de varios miembros de la familia, etc.

Los gitanos, como afirma Teresa San Román, prefieren seguir al margen, ocupando trabajos "marginales", cuando lo que les ofrece la sociedad es menos de lo que consiguen con ese tipo de ocupaciones. En este sentido, se debe tener en cuenta que la mayor parte de los gitanos a la hora de optar entre dos trabajos no valoran únicamente la cantidad económica que se percibe, sino que le dan gran importancia a otros aspectos cualitativos, del tipo de los señalados.

Con la crisis económica de la segunda mitad de los años setenta, se produjeron reducciones de plantilla en un gran número de empresas, y los primeros en ir a la calle fueron precisamente aquellos que entraron más tarde y que ocupaban los puestos más bajos, y es en ese grupo donde se incluían los gitanos. (En cualquier caso, no quisiéramos dar una imagen homogénea de los gitanos y no obviamos que ha habido diversas situaciones en base a distintas variables).

9 Sobre los trabajos realizados por los gitanos durante las décadas de los años sesenta y setenta, v. EQUIPO GIEMS, Los gitanos al encuentro de la ciudad: Del chalaneo al peonaje. Edicusa. Madrid, 1976. 


\section{EL MITO DEL GITANO «ERRANTE»}

Con respecto a las migraciones gitanas, hay que decir que hace ya muchos años que el gitano español errante es más un mito que una realidad. Es cierto que hasta nuestras fechas se ha mantenido para muchas familias gitanas unas migraciones periódicas con motivos tanto laborales como sociales. Muchas familias se trasladan para trabajar un par de meses al año en la recolección de fruta, u otros trabajos determinados, o bien visitar a sus familiares, (que pueden residir en lugares lejanos), durante las navidades, San Juan, y Los Santos, y ésas alargarse durante más de una semana. Sin embargo, son migraciones con un lugar fijo de partida y retorno. Este seminomadismo parece que hace ya siglos que se realizaba y ha estado muy relacionado con los distintos oficios practicados por los gitanos, como la venta por los pueblos, los arreglos de cacharrerías, etc.

En la actualidad, el seminomadismo de los gitanos españoles, si bien se sigue manteniendo en algunas familias, lo cierto es que ha disminuido de forma importante. Entre los factores que han provocado esa disminución del viaje (aunque fuese temporal), están:

1. La mayor parte de los niños gitanos están escolarizados, y supone un lastre sacarlos del colegio durante largo tiempo (máxime cuando se recibe algún tipo de ayuda condicionada a la escolarización de los hijos).

2. Algunos gitanos tienen trabajos asalariados que les suponen tener un horario y unos días de trabajo rígidos, por lo que no pueden ausentarse demasiado tiempo.

3. El seminomadismo realizado hasta ahora se realizaba en muchos casos para dedicarse a la venta ambulante, recorriendo las distintas poblaciones por donde pasaban con sus artículos. Pero en la actualidad este tipo de venta está prohibida en la mayoría de los puebles, estableciéndose unos días de venta y una considerable burocracia para obtener un puesto en el mercado.

4. Con la aparición de los vehículos a motor, los viajes se pueden realizar en horas, y no en días como antaño, con lo que una familia puede ir a vender a una ciudad que se encuentre a $200 \mathrm{kms}$. y volver en el mismo día. De la misma forma que se puede visitar a la familia y volver en el mismo fin de semana.

5. La dependencia que algunas familias tienen de las ayudas de Servicios Sociales les ata a un sitio determinado. Como decíamos en el caso del colegio de los niños, o de determinadas contraprestaciones, cursos, etc., el ausentarse durante un tiempo más o menos amplio (injustificado desde la óptica etnocentrista de las instituciones "payas"), puede suponer perder una ayuda económica, la casa de realojo, etc. 
Por otro lado, las emigraciones de gitanos españoles al extranjero ya apenas se practican, y se centraron principalmente en los viajes a Centro y Sudamérica, donde se dedicaban a la venta, y al sur de Francia, donde realizaban tareas de recolección en la época de la Vendimia.

\section{LOS GITANOS ANTE LAS NUEVAS CORRIENTES MIGRA- TORIAS: COMPETENCIA ÍNTER E INTRA-ÉTNICA}

Desde los años setenta comienza una inmigración de gitanos portugueses que llegan en primer lugar a las zonas de Galicia y Extremadura, y que más tarde irían recorriendo todo el país. Se trata en la mayoría de los casos de personas con escasísimos recursos económicos que van recorriendo ciudades, sin ningún tipo de arraigo, y que en la mayor parte de los casos acaban formando parte de los barrios chabolistas que se encuentran en la periferia de muchas ciudades ${ }^{10}$.

Por otro lado, desde la caída del Muro de Berlín, hay un éxodo de familias gitanas que huyen de la miseria, la persecución política y, en el caso yugoeslavo, la guerra.

Es de resaltar el caso de los gitanos de la ex-Checoeslovaquia, donde a millares de gitanos residentes en estas dos repúblicas se les niega ahora cualquiera de las dos nacionalidades ${ }^{11}$.

Este flujo de gitanos del Este recorre Europa y empiezan a llegar también a España, retomando el camino errante de nuestras carreteras que hace ya años abandonaron los gitanos españoles.

A estas inmigraciones gitanas de Portugal y del Este de Europa se sumaron los nuevos inmigrantes procedentes de los distintos países del sur (Magreb, África negra, Sudamérica, etc.). Así, el gran número de gitanos que no habían conseguido con la Revolución Industrial mejorar su situación ni ser incluidos en una sociedad mayoritaria que no parece admitir las diferencias (y una vez más recordamos que no son todos los gitanos españoles) aquellos gitanos que hoy siguen viviendo de una economía marginal se encuentran ahora con una doble competencia:

- Intra-étnica,

- Ínter-étnica.

La competencia intra-étnica se produce con aquellos gitanos que llegan de Portugal y del Este de Europa, compitiendo en muchos casos por los mismos recursos (entre ellos los Servicios Sociales) y dando en ocasiones una imagen del gitano que no gusta a los gitanos españoles.

10 Colectivo IOE. "Los inmigrantes en España" en Documentación Social. Revista de Estudios Sociales y de Sociología aplicada. Cáritas Española. Madrid, 1987.

"I ACNUR: "Refugiados en Europa Central", en Revista Papeles. pp. 73-89. 
Curiosamente, no parece producirse con estas familias la tradicional solidaridad entre gitanos de la que a veces se hace gala, ya que este tipo de solidaridad se suele dar dentro de las familias extensas o ante un conflicto con el grupo mayoritario. Por otro lado, estas familias pueden ser vistas en ocasiones como un peligro de conflicto entre familias residentes en un barrio.

Por otro lado, la competencia ínter-étnica se produce con los inmigrantes de los llamados «Países del sur» que llegan en situaciones económicamente precarias, dispuestos a trabajar bajo situaciones laborales infrahumanas muchas veces sin un permiso de residencia que les permitiera acceder a un trabajo regularizado, por lo que se encuentran en el margen y por tanto compitiendo por una serie de actividades laborales marginales, que tradicionalmente han sido desempeñadas por un sector importante del colectivo gitano, como recogida de chatarra, venta en mercadillos, etc.

En esta pugna de carácter competitivo se dan enfrentamientos, ya que existen determinados aspectos comunes que se traducen en la práctica como un aumento de los conflictos entre las clases más desfavorecidas. La inseguridad sobre su futuro, las condiciones de vida infrahumanas, la marginación socio-cultural, la xenofobia sufrida por ambos colectivos, son algunas de las variables que favorecen esta problemática. Asimismo, los estudios realizados por T. Calvo Buezas ${ }^{12}$, en nuestro país, demuestran que los gitanos continúan estando peor considerados, sean de donde sean, que cualquier inmigrante, de ahí que en ocasiones un determinado trabajo o puesto de venta se otorgue a un magrebí, por ejemplo, que a un gitano, lo que aumenta la tensión ínter-étnica.

En este apartado de competencia por los recursos marginales, caben resaltar la visión e influencia de las ayudas de Servicios Sociales, en este sentido se atienden necesidades de vivienda, ropa, alimentación, salud, ayuda para alquileres, informes para contratos de trabajo, etc. El aumento de inmigrantes legales que son derivados a estos servicios favorece también al conflicto con una parte de la comunidad gitana, existiendo una lucha por los recursos sociales que actualmente son escasos.

\section{CONCLUSIONES PARA UN FUTURO}

Después de este breve recorrido histórico y actual del pueblo gitano, como una larga trayectoria de inmigración hacia Europa y al Occi-

12 CALVO BUEZAS, Tomás: Crece el racismo, también la solidaridad. Junta de Extremadura. Ed. Tecnos. Madrid, 1995. p. 44. 
dente, nos gustaría hacer una reflexión sobre el futuro, no tan lejano. Desde las diversas ópticas que podríamos enfocar este problema, quisiéramos hacerlo desde la positiva.

Aunque el problema de España como receptora de inmigrantes de África y filtro de Europa, entre el Sur pobre y el Norte rico, los movimientos migratorios van a ser más intensos, quedando muchos grupos de estas personas atrapados en nuestro país a la espera de la documentación que les legalice y por la política de cupos para inmigrantes, permaneciendo en una situación de desamparo en todos los aspectos y siendo cada vez más rechazados por una sociedad, que se llama «solidaria», pero que es recelosa de que nos quiten lo que tenemos, reforzada por la política europea de cierre de fronteras a los países pobres.

Nuestra aportación como profesionales, ante este orden social, económico y político, creemos que debe de ir dirigido a la potenciación de programas y medidas para la sensibilización y concienciación de la sociedad mayoritaria, a través de estudios rigurosos de las características y peculiaridades culturales, de los diferentes grupos étnicos que conviven en la sociedad española.

Estos estudios nos ayudarían a reflexionar y comprender determinados aspectos, que, como sabemos, están impidiendo que se dé una verdadera "integración", adaptada a esas características culturales de cada individuo en su momento determinado, así como a proponer alternativas de solución eficaces a sus problemas y necesidades, favoreciendo un trabajo desde la tolerancia y el respeto a la diferencia, como para proporcionar una información más veraz sobre estos colectivos, que sea la base para ir eliminando actitudes prejuiciosas, que están vigentes en algunos de los profesionales que trabajamos con esta población.

La escasez de recursos económicos, sociales..., la aparición de una mayor demanda de los mismos, los brotes de racismo resurgidos contra estas colectividades en toda Europa, la confrontación entre e intra etnias, el desconocimiento mutuo, la crisis económica que provoca desplazamientos masivos desde el denominado tercer mundo al también denominado primer mundo, la pobreza extrema sufrida por los colectivos marginales, y su exclusión social, nos están planteando un reto; si queremos convivir en una sociedad plural, enriqueciéndonos, con el tan manido y mal utilizado concepto de «INTERCULTURALIDAD», que, en muchas ocasiones, produce el efecto contrario, es decir, asimilación y fagotización a las normas y costumbres mayoritarias, en detrimento de una respeto a las diferentes.

En este proceso, la potenciación de campañas de información por parte de la administración y de las distintas organizaciones no gubernamentales, así como las aportaciones de los medios de comunicación, son imprescindibles para conocer las diversidades, así existiría una 
verdadera convivencia basada en el respeto y enriquecimiento personal y social en todos los ámbitos de la vida.

\section{BIBLIOGRAFIA :}

ACNUR: "Refugiados en Europa Central", en Revista Papeles. pp. 73-89.

BLAY, F. et alt.: Propuesta para un trabajo intercultural en la escuela. Fichas de trabajo. Valencia, 1989.

CALVO BUEZAS, Tomás: Crece el racismo, también la solidaridad. Junta de Extremadura. Ed. Tecnos. Madrid, 1995. p. 44.

Colectivo IOE: "Los inmigrantes en España" en Documentación Social. Revista de Estudios Sociales y de Sociología aplicada. Cáritas Española. Madrid, 1987.

EQUIPO CLAVES: Ponencia:"El discurso de las Organizaciones no Gubernamentales y de las Instituciones públicas ante la población inmigrante". Curso de iniciación a los Derechos Hunanos: Los Derechos Humanos ante el racismo y la xenofobía. Cabueñes 1.995 .

EQUIPO GIEMS: Los gitanos al encuentro de la ciudad: Del chalaneo al peonaje. Edicusa. Madrid, 1976.

GÓMEZ ALFARO, Antonio: La gran redada de los gitanos. Coleción Interface. Centro de investigaciones gitanas. Ed. Presencia Gitana. Madrid ,1.993

HIDALGO TUÑÓN, Alberto: Reflexión ética sobre el Racismo y la Xenofobia. Fundamentos teóricos. Ed. Popular.Jóvenes Contra la Intolerancia. Madrid 1.994.

LIGEOIS, B.: Gitanos e itinerantes. Madrid, 1.987

SAN ROMAN, T.: Vecinos gitanos. Madrid, 1976.

SAN ROMÁN, T.: La diferència inquietant: velles i noves estratégies culturals dels gitanos. Alta Fulla. BCN, 1994.

SÁNCHEZ ORTEGA, Mํㅡㄹ Elena y SAN ROMÁN, Teresa: Entre la marginación y el racismo. Reflexiones sobre la vida de los gitanos. Madrid, 1986.

"I Tchatchipen": Publicación trimestral de investigación gitana. Ed. Instituto Romano de Servicios Sociales y Culturales. 